\title{
Shear viscosity for a heated granular binary mixture at low-density
}

\author{
José María Montanero* \\ Departamento de Electrónica e Ingeniería Electromecánica, Universidad de Extremadura, \\ E-06071 Badajoz, Spain \\ Vicente Garzó ${ }^{\dagger}$ \\ Departamento de Física, Universidad de Extremadura, E-06071 Badajoz, Spain
}

(November 5, 2018)

\begin{abstract}
The shear viscosity for a heated granular binary mixture of smooth hard spheres at low-density is analyzed. The mixture is heated by the action of an external driving force (Gaussian thermostat) which exactly compensate for cooling effects associated with the dissipation of collisions. The study is made from the Boltzmann kinetic theory, which is solved by using two complementary approaches. First, a normal solution of the Boltzmann equation via the Chapman-Enskog method is obtained up to first order in the spatial gradients. The mass, heat, and momentum fluxes are determined and the corresponding transport coefficients identified. As in the free cooling case [V. Garzó and J. W. Dufty, Phys. Fluids 14, 1476 (2002)], practical evaluation requires a Sonine polynomial approximation, and here it is mainly illustrated in the case
\end{abstract}

\footnotetext{
*Electronic address: jmm@unex.es

†Electronic address: vicenteg@unex.es
} 
of the shear viscosity. Second, to check the accuracy of the Chapman-Enskog results, the Boltzmann equation is numerically solved by means of the Direct Simulation Monte Carlo (DSMC) method. The simulation is performed for a system under uniform shear flow, using the Gaussian thermostat to control inelastic cooling. The comparison shows an excellent agreement between theory and simulation over a wide range of values of the restitution coefficients and the parameters of the mixture (masses, concentrations, and sizes).

PACS number(s): 45.70.Mg, 05.20.Dd, 51.10.+y

Typeset using REVTEX 


\section{INTRODUCTION}

The macroscopic behavior of rapid granular flows can be described through hydrodynamic equations accounting for dissipation among the interacting particles. A basis for the derivation of the hydrodynamic equations and explicit expressions for the transport coefficients appearing in them is provided by the corresponding Boltzmann kinetic theory in the low-density regime. In the simplest model the grains are taken to be smooth hard spheres with inelastic collisions. Assuming the existence of a normal solution for sufficiently long space and time scales, the Chapman-Enskog method [1] can be applied to get the velocity distribution function in terms of the hydrodynamic fields and their spatial gradients. This method must be conveniently adapted to inelastic collisions due to the new time dependence of temperature resulting from collisional energy loss. In the case of a monocomponent gas, the Navier-Stokes transport coefficients have been obtained in terms of the restitution coefficient without limitation on the degree of inelasticity [2-4]. This analysis for a monocomponent system has been also extended to dense gases in the context of the Enskog equation [5].

Similar studies for multicomponent granular gases are more scarce and most of them limited to the asymptotically weak dissipation limit [6]. Although these studies permit in principle different temperatures for each species, they usually assume energy equipartition and so the partial temperatures $T_{i}$ are made equal to the mixture temperature $T$. However, some recent results obtained in molecular dynamics simulations [7] as well as in real experiments of vibrated mixtures in three [8] and two [9] dimensions clearly show the breakdown of energy equipartition. A more recent kinetic theory calculation which takes into account temperature differences has been carried out by Garzó and Dufty [10]. They solved the set of Boltzmann coupled equations of the binary mixture by means of the Chapman-Enskog expansion for states near the local homogeneous cooling state. The mass, heat, and momentum fluxes were determined to first order in the gradients of the hydrodynamic fields and the associated transport coefficients were explicitly identified. As in the case of elastic collisions 
[1], these transport coefficients verify a set of coupled linear integral equations which are solved approximately by using the leading terms in a Sonine polynomial expansion. The results derived by Garzó and Dufty [10] provide a description of hydrodynamics in binary granular mixtures valid a priori over the broadest parameter range and not limited to the quasielastic regime. In particular, the consequences of the temperature differences on the transport coefficients were shown to be quite significant.

In the case of molecular fluid mixtures, it is known that the leading order truncation is quite accurate, except for extreme mass ratios (e.g., electron-proton systems). Much less is known in the case of inelastic collisions, although some comparisons with computer simulations for homogeneous states indicate that the accuracy is similar to that for elastic collisions $[11,12]$. The objective here is to compare the kinetic theory predictions for the shear viscosity with those obtained from a numerical solution of the Boltzmann equation by means of the Direct Simulation Monte Carlo (DSMC) method [13]. Specifically, the simulations are performed for a granular mixture undergoing uniform shear flow (USF), namely, a macroscopic state characterized by constant partial densities $n_{i}(i=1,2)$, uniform temperature $T$ and a linear flow velocity profile $u_{i, x}=u_{x}=a y, a$ being the constant shear rate. In a molecular fluid under USF, the temperature increases in time due to viscous heating. As a consequence, the average collision frequency $\nu$ (which is proportional to $T^{1 / 2}$ for hard spheres) increases with time and the reduced shear rate $a^{*}=a / \nu$ (which is the relevant nonequilibrium parameter of the problem) tends to zero in the long time limit. This implies that for sufficiently long times the system reaches a regime described by linear hydrodynamics and the Navier-Stokes shear viscosity can be identified $[14,15]$. For granular fluids, the inelasticity of collisions introduces an energy sink in the balance equation for the temperature. Thus, the relationship between the temperature and the shear viscosity is not as simple as for molecular fluids since there is a competition between viscous heating and collisional cooling. However, if the granular fluid is externally excited by an external energy source that exactly compensates for the collisional energy loss, the viscous heating effect is still able to heat the system (as in the elastic case) and one can 
identify the shear viscosity in the limit of small shear rate relative to the collision frequency $\nu$ (i.e., $\left.a^{*} \rightarrow 0\right)$. Although there are several choices for the external driving force, here we consider an external thermostat proportional to the peculiar velocity. This thermostat has been frequently used in nonequilibrium molecular dynamics simulations of molecular fluids [16] and has the advantage that, in the absence of shear, it does not affect the dynamics of the system at all since it is formally equivalent to a rescaling of the velocities [7] .

The motivation of our study is twofold. First, in light of some doubts about the validity of a hydrodynamic description for granular flow, the comparison with simulation allows us to test the Chapman-Enskog solution obtained by assuming the existence of a normal or hydrodynamic regime. Since the parameter space here is quite large the tests of the theory and concepts are quite stringent. Second, as said above, we can also assess the degree of reliability of the approximate solution (first Sonine polynomial approximation) to the resulting integral equation over a wide range of the parameter space. With respect to the driving external force used in our analysis, we do not claim that it is the most suited one to model any real experiment. However, it has the advantage that it can be incorporated into kinetic theory and computer simulations very easily and it allows to check the assumptions of the Chapman-Enskog method.

The plan of the paper is as follows. In Section II, we review the Boltzmann equation and associated macroscopic conservation laws in the presence of the Gaussian thermostat. The Chapman-Enskog method is applied in Section III to get all the transport coefficients of the mixture, with special emphasis in the shear viscosity coefficient. The details of the derivation are displayed in Appendix A. Section IV deals with the application of the DSMC method of the Boltzmann equation to USF with thermostat. The Chapman-Enskog and simulation results are compared in Section $\mathrm{V}$ at the level of the shear viscosity showing a good agreement. We close the paper in Section VI with a discussion of the results presented. 


\section{THE BOLTZMANN EQUATION AND TRANSPORT COEFFICIENTS}

We consider a binary mixture of smooth hard spheres of masses $m_{1}$ and $m_{2}$, and diameters $\sigma_{1}$ and $\sigma_{2}$. The inelasticity of collisions among all pairs is characterized by three independent constant coefficients of normal restitution $\alpha_{11}, \alpha_{22}$, and $\alpha_{12}=\alpha_{21}$, where $\alpha_{i j}$ is the restitution coefficient for collisions between particles of species $i$ and $j$. Due to the intrinsic dissipative character of collisions, an energy supply is requested to fluidize a granular gas. For simplicity, here the fluidization is driven by the action of a non-conservative external force, frequently referred to as the Gaussian thermostat. In this case, the mixture is heated by an "anti-drag" force, linear in the peculiar velocity $\mathbf{V}$ and chosen to exactly compensate for collisional cooling. As said in the Introduction, this deterministic thermostat has been widely used in computer simulations of molecular fluids [16]. Under these conditions and in the low-density regime, the distribution functions $f_{i}(\mathbf{r}, \mathbf{v} ; t)(i=1,2)$ for the two species are determined from the set of nonlinear Boltzmann equations

$$
\left(\partial_{t}+\mathbf{v}_{1} \cdot \nabla\right) f_{i}+\frac{1}{2} \xi \frac{\partial}{\partial \mathbf{v}_{1}} \cdot\left(\mathbf{V}_{1} f_{i}\right)=\sum_{j} J_{i j}\left[\mathbf{v}_{1} \mid f_{i}(t), f_{j}(t)\right]
$$

where the constant $\xi$ is taken to be the same for each species $[7,12,17]$. Here, $\mathbf{V}_{1} \equiv \mathbf{v}_{1}-\mathbf{u}$, $\mathbf{u}$ being the flow velocity. The Boltzmann collision operator $J_{i j}\left[\mathbf{v}_{1} \mid f_{i}, f_{j}\right]$ describing the scattering of pairs of particles is

$$
\begin{aligned}
J_{i j}\left[\mathbf{v}_{1} \mid f_{i}, f_{j}\right]= & \sigma_{i j}^{2} \int d \mathbf{v}_{2} \int d \widehat{\boldsymbol{\sigma}} \Theta\left(\widehat{\boldsymbol{\sigma}} \cdot \mathbf{g}_{12}\right)\left(\widehat{\boldsymbol{\sigma}} \cdot \mathbf{g}_{12}\right) \\
& \times\left[\alpha_{i j}^{-2} f_{i}\left(\mathbf{r}, \mathbf{v}_{1}^{\prime}, t\right) f_{j}\left(\mathbf{r}, \mathbf{v}_{2}^{\prime}, t\right)-f_{i}\left(\mathbf{r}, \mathbf{v}_{1}, t\right) f_{j}\left(\mathbf{r}, \mathbf{v}_{2}, t\right)\right],
\end{aligned}
$$

where $\sigma_{i j}=\left(\sigma_{i}+\sigma_{j}\right) / 2, \hat{\boldsymbol{\sigma}}$ is a unit vector along their line of centers, $\Theta$ is the Heaviside step function, and $\mathbf{g}_{12}=\mathbf{v}_{1}-\mathbf{v}_{2}$. The primes on the velocities denote the initial values $\left\{\mathbf{v}_{1}^{\prime}, \mathbf{v}_{2}^{\prime}\right\}$ that lead to $\left\{\mathbf{v}_{1}, \mathbf{v}_{2}\right\}$ following a binary collision:

$$
\mathbf{v}_{1}^{\prime}=\mathbf{v}_{1}-\mu_{j i}\left(1+\alpha_{i j}^{-1}\right)\left(\widehat{\boldsymbol{\sigma}} \cdot \mathbf{g}_{12}\right) \hat{\boldsymbol{\sigma}}, \quad \mathbf{v}_{2}^{\prime}=\mathbf{v}_{2}+\mu_{i j}\left(1+\alpha_{i j}^{-1}\right)\left(\widehat{\boldsymbol{\sigma}} \cdot \mathbf{g}_{12}\right) \widehat{\boldsymbol{\sigma}},
$$

where $\mu_{i j}=m_{i} /\left(m_{i}+m_{j}\right)$. 
The relevant hydrodynamic fields are the number densities $n_{i}$, the flow velocity $\mathbf{u}$, and the "granular" temperature $T$. They are defined in terms of moments of the distributions $f_{i}$ as

$$
\begin{gathered}
n_{i}=\int d \mathbf{v} f_{i}(\mathbf{v}), \quad \rho \mathbf{u}=\sum_{i} \int d \mathbf{v} m_{i} \mathbf{v} f_{i}(\mathbf{v}) \\
n T=p=\sum_{i} \int d \mathbf{v} \frac{m_{i}}{3} V^{2} f_{i}(\mathbf{v})
\end{gathered}
$$

where $n=n_{1}+n_{2}$ is the total number density, $\rho=m_{1} n_{1}+m_{2} n_{2}$ is the total mass density, and $p$ is the hydrostatic pressure. At a kinetic level, it is convenient to introduce the kinetic temperatures $T_{i}$ for each species defined as

$$
\frac{3}{2} n_{i} T_{i}=\int d \mathbf{v} \frac{m_{i}}{2} V^{2} f_{i}
$$

The collision operators conserve the particle number of each species and the total momentum but the total energy is not conserved:

$$
\begin{gathered}
\int d \mathbf{v} J_{i j}\left[\mathbf{v} \mid f_{i}, f_{j}\right]=0, \quad \sum_{i, j} \int d \mathbf{v} m_{i} \mathbf{v} J_{i j}\left[\mathbf{v} \mid f_{i}, f_{j}\right]=0, \\
\sum_{i, j} \int d \mathbf{v} \frac{1}{2} m_{i} \mathbf{v}^{2} J_{i j}\left[\mathbf{v} \mid f_{i}, f_{j}\right]=-\frac{3}{2} n T \zeta
\end{gathered}
$$

where $\zeta$ is identified as the cooling rate due to inelastic collisions among all species. The macroscopic balance equations follow from the Boltzmann equation (1) and Eqs. (7) and (8). They are given by

$$
\begin{gathered}
D_{t} n_{i}+n_{i} \nabla \cdot \mathbf{u}+\frac{\nabla \cdot \mathbf{j}_{i}}{m_{i}}=0, \\
D_{t} \mathbf{u}+\rho^{-1} \nabla \mathrm{P}=0, \\
D_{t} T-\frac{T}{n} \sum_{i} \frac{\nabla \cdot \mathbf{j}_{i}}{m_{i}}+\frac{2}{3 n}(\nabla \cdot \mathbf{q}+\mathrm{P}: \nabla \mathbf{u})=-(\zeta-\xi) T .
\end{gathered}
$$

In the above equations, $D_{t}=\partial_{t}+\mathbf{u} \cdot \nabla$ is the material derivative, 


$$
\mathbf{j}_{i}=m_{i} \int d \mathbf{v} \mathbf{V} f_{i}(\mathbf{v})
$$

is the mass flux for species $i$ relative to the local flow,

$$
\mathbf{P}=\sum_{i} \int d \mathbf{v} m_{i} \mathbf{V} \mathbf{V} f_{i}(\mathbf{v})
$$

is the total pressure tensor, and

$$
\mathbf{q}=\sum_{i} \int d \mathbf{v} \frac{1}{2} m_{i} V^{2} \mathbf{V} f_{i}(\mathbf{v})
$$

is the total heat flux.

The energy balance equation (11) shows that the existence of a driving with the choice $\xi=\zeta$ compensates for the cooling effect due to the inelasticity of collisions. In that case, the macroscopic balance equations look like those of a conventional mixture with elastic collisions. Nevertheless, the transport coefficients entering in the constitutive equations are in general different from those of a gas of elastic particles. Furthermore, for systems with elastic collisions, the specific set of gradients contributing to each flux is restricted by fluid symmetry, time reversal invariance (Onsager relations), and the form of the entropy porduction [18]. In the case of inelastic collisions only fluid symmetry applies and so there is more flexibility in representing the fluxes and identifying the corresponding transport coefficients. It follows from fluid symmetry that the pressure tensor has the same form to first order in the gradients as for the monocomponent gas. In the case of heat and mass fluxes, several different (but equivalent) choices of hydrodynamic fields can be used and some care is required in comparing transport coefficients in the different representations. Here, as done in the unforced case [10], we take the gradients of the mole fraction $x_{1}=n_{1} / n$, the pressure $p$, the temperature $T$ and the flow velocity $\mathbf{u}$ as the relevant ones. Thus, in this representation, the phenomenological constitutive relations for the fluxes in the low-density regime have the forms [19]

$$
\begin{gathered}
\mathbf{j}_{1}=-\frac{m_{1} m_{2} n}{\rho} D \nabla x_{1}-\frac{\rho}{p} D_{p} \nabla p-\frac{\rho}{T} D^{\prime} \nabla T, \quad \mathbf{j}_{2}=-\mathbf{j}_{1} \\
\mathbf{q}=-T^{2} D^{\prime \prime} \nabla x_{1}-L \nabla p-\lambda \nabla T
\end{gathered}
$$




$$
P_{\alpha \beta}=p \delta_{\alpha \beta}-\eta\left(\nabla_{\beta} u_{\alpha}+\nabla_{\alpha} u_{\beta}-\frac{2}{3} \delta_{\alpha \beta} \nabla \cdot \mathbf{u}\right)
$$

The transport coefficients are the diffusion coefficient $D$, the thermal diffusion coefficient $D^{\prime}$, the pressure diffusion coefficient $D_{p}$, the Dufour coefficient $D^{\prime \prime}$, the thermal conductivity $\lambda$, the pressure energy coefficient $L$, and the shear viscosity $\eta$. The Chapman-Enskog method [1] generalized to inelastic collisions allows one to get explicit expressions for these transport coefficients as functions of the restitution coefficients and the parameters of the mixture.

\section{SHEAR VISCOSITY OF A HEATED GRANULAR MIXTURE}

The Chapman-Enskog method assumes the existence of a normal solution in which all space and time dependence of the distribution function occurs through a functional dependence on the hydrodynamic fields

$$
f_{i}\left(\mathbf{r}, \mathbf{v}_{1}, t\right)=f_{i}\left[\mathbf{v}_{1} \mid x_{1}(\mathbf{r}, t), p(\mathbf{r}, t), T(\mathbf{r}, t), \mathbf{u}(\mathbf{r}, t)\right]
$$

This functional dependence can be made local in space and time by means of an expansion in gradients of the fields. Thus, we write $f_{i}$ as a series expansion in a formal parameter $\epsilon$ measuring the nonuniformity of the system,

$$
f_{i}=f_{i}^{(0)}+\epsilon f_{i}^{(1)}+\epsilon^{2} f_{i}^{(2)}+\cdots,
$$

where each factor of $\epsilon$ means an implicit gradient of a hydrodynamic field. The local reference state $f_{i}^{(0)}$ is chosen to give the same first moments as the exact distribution $f_{i}$. The time derivatives of the fields are also expanded as $\partial_{t}=\partial_{t}^{(0)}+\epsilon \partial_{t}^{(1)}+\cdots$. The coefficients of the time derivative expansion are identified from the balance equations (9)-(11) after expanding the fluxes, and the cooling rate $\zeta$ in a similar series as (19). More details on the ChapmanEnskog method adapted to inelastic collisions can be found in Ref. [10]. Now, the main difference with respect to the free cooling case [10] is that the sink term in the energy equation is zero (when one takes $\xi=\zeta$ ), so that the terms coming from the time derivative $\partial_{t}^{(0)}$ vanish. 
In the zeroth order, $f_{i}^{(0)}$ obeys the kinetic equation

$$
\frac{1}{2} \zeta^{(0)} \frac{\partial}{\partial \mathbf{V}} \cdot\left(\mathbf{V} f_{i}^{(0)}\right)=\sum_{j} J_{i j}\left[f_{i}^{(0)}, f_{j}^{(0)}\right]
$$

where use has been made of the choice $\xi^{(0)}=\zeta^{(0)}$. Here, $\zeta^{(0)}$ is determined by Eq. (8) to zeroth order. With this choice, Eq. (20) is identical to the the one obtained in the unforced case [20], and there is an exact correspondence between the homogeneous cooling state and this type of driven steady state. This is one of the advantages of the Gaussian thermostat. Dimensional analysis requires that $f_{i}^{(0)}(\mathbf{V})$ must be of the form

$$
f_{i}^{(0)}(V)=n_{i} v_{0}^{-3} \Phi_{i}\left(V / v_{0}\right)
$$

where

$$
v_{0}=\sqrt{2 T \frac{m_{1}+m_{2}}{m_{1} m_{2}}}
$$

is a thermal velocity defined in terms of the temperature $T$ of the mixture. So far, the exact form of $\Phi_{i}$ has not been found, although a good approximation for thermal velocities can be obtained from an expansion in Sonine polynomials [20]. In the leading order, $\Phi_{i}$ is given by

$$
\Phi_{i}\left(V^{*}\right) \rightarrow\left(\frac{\theta_{i}}{\pi}\right)^{3 / 2} e^{-\theta_{i} V^{* 2}}\left[1+\frac{c_{i}}{4}\left(\theta_{i} V^{* 4}-5 \theta_{i} V^{* 2}+\frac{15}{4}\right)\right],
$$

where $V^{*}=V / v_{0}, \theta_{i}=\left(\mu_{j i} \gamma_{i}\right)^{-1}$, and $\gamma_{i}=T_{i} / T$. The coefficients $c_{i}$ (which measure the deviation of $\Phi_{i}$ from the reference Maxwellian) are determined consistenly from the Boltzmann equation. The approximation (23) provides detailed predictions for the cooling rate $\zeta^{(0)}$, the temperature ratio $T_{1} / T_{2}$ and the cumulants $c_{i}$ as functions of the mass ratio, size ratio, composition and restitution coefficients. Recently, the accuracy of this approximate solution has been confirmed by Monte Carlo simulation of the Boltzmann equation over a wide range of the parameter space [11] .

The analysis to first order in $\epsilon$ is similar to the one worked out in Ref. [10] for the free cooling case. Some details of the derivation of the transport coefficients as well as their final expressions are given in Appendix A. Here, given that the theoretical predictions for 
the shear viscosity coefficient $\eta$ will be compared with those obtained from Monte Carlo simulations, we focus our attention on the explicit final expression for $\eta$. According to Eq. (A25), the shear viscosity can be written as

$$
\eta=\frac{n T}{\nu} \eta^{*}
$$

where

$$
\nu=\sqrt{\pi} n \sigma_{12}^{2} v_{0}
$$

is an effective collision frequency and the reduced shear viscosity coefficient $\eta^{*}$ is

$$
\eta^{*}=x_{1} \gamma_{1}^{2} d_{1,1}^{*}+x_{2} \gamma_{2}^{2} d_{2,1}^{*}
$$

with

$$
d_{1,1}^{*}=\frac{\gamma_{2}\left(\tau_{22}^{*}-\zeta^{*}\right)-\gamma_{1} \tau_{12}^{*}}{\Delta}, \quad d_{2,1}^{*}=\frac{\gamma_{1}\left(\tau_{11}^{*}-\zeta^{*}\right)-\gamma_{2} \tau_{21}^{*}}{\Delta} .
$$

Here, $\zeta^{*}=\zeta^{(0)} / \nu$,

$$
\Delta=\gamma_{1} \gamma_{2}\left[\zeta^{* 2}-\zeta^{*}\left(\tau_{11}^{*}+\tau_{22}^{*}\right)+\tau_{11}^{*} \tau_{22}^{*}-\tau_{12}^{*} \tau_{21}^{*}\right]
$$

and the dimensionless quantities $\tau_{i j}^{*}$ are given by [10]

$$
\begin{aligned}
\tau_{11}^{*}= & \frac{16}{5 \sqrt{2}} x_{1}\left(\frac{\sigma_{1}}{\sigma_{12}}\right)^{2} \theta_{1}^{-1 / 2}\left[1-\frac{1}{4}\left(1-\alpha_{11}\right)^{2}\right]\left(1-\frac{c_{1}}{64}\right) \\
& +\frac{8}{15} x_{2} \mu_{21}\left(1+\alpha_{12}\right) \theta_{1}^{3 / 2} \theta_{2}^{-1 / 2}\left[6 \theta_{1}^{-2}\left(\mu_{12} \theta_{2}-\mu_{21} \theta_{1}\right)\left(\theta_{1}+\theta_{2}\right)^{-1 / 2}\right. \\
& +\frac{3}{2} \mu_{21} \theta_{1}^{-2}\left(\theta_{1}+\theta_{2}\right)^{1 / 2}\left(3-\alpha_{12}\right)+5 \theta_{1}^{-1}\left(\theta_{1}+\theta_{2}\right)^{-1 / 2} \\
& \left.+\frac{c_{2}}{16} \frac{2 \theta_{2}\left(12 \mu_{21}+9 \mu_{12}-10\right)-\theta_{1}\left(5-6 \mu_{21}\right)-\frac{3}{2} \mu_{21}\left(3-\alpha_{12}\right)\left(\theta_{1}+\theta_{2}\right)}{\left(\theta_{1}+\theta_{2}\right)^{5 / 2}}\right], \\
\tau_{12}^{*}= & \left.\frac{8}{15} x_{2} \frac{\mu_{21}^{2}\left(1+\alpha_{12}\right) \theta_{1}^{3 / 2} \theta_{2}^{-1 / 2}\left[6 \theta_{2}^{-2}\left(\mu_{12} \theta_{2}-\mu_{21} \theta_{1}\right)\left(\theta_{1}+\theta_{2}\right)^{-1 / 2}\right.}{\mu_{12}}\right] \\
& +\frac{3}{2} \mu_{21} \theta_{2}^{-2}\left(\theta_{1}+\theta_{2}\right)^{1 / 2}\left(3-\alpha_{12}\right)-5 \theta_{2}^{-1}\left(\theta_{1}+\theta_{2}\right)^{-1 / 2} \\
& \left.+\frac{c_{1}}{16} \frac{2 \theta_{1}\left(10-12 \mu_{12}-9 \mu_{21}\right)+\theta_{2}\left(5-6 \mu_{12}\right)-\frac{3}{2} \mu_{21}\left(3-\alpha_{12}\right)\left(\theta_{1}+\theta_{2}\right)}{\left(\theta_{1}+\theta_{2}\right)^{5 / 2}}\right] .
\end{aligned}
$$


The corresponding expressions for $\tau_{22}^{*}$ and $\tau_{21}^{*}$ can be inferred from Eqs. (29) and (30) by interchanging $1 \leftrightarrow 2$.

Equations (24)-(30) provide the explicit expression for the shear viscosity $\eta$ of a heated granular binary mixture in the first Sonine approximation. It is apparent that the reduced viscosity $\eta^{*}$ presents a complex nonlinear dependence on the restitution coefficients $\alpha_{11}$, $\alpha_{22}$, and $\alpha_{12}$ and the parameters of the mixture $m_{1} / m_{2}, \sigma_{1} / \sigma_{2}$, and $n_{1} / n_{2}$. The quality of the expression for $\eta^{*}$ will be later assessed by comparison with Monte Carlo simulations in the uniform shear flow (USF) problem with a thermostat. Before studying the general dependence of $\eta^{*}$ on the parameter space, it is instructive to consider some special limit cases. In the elastic limit, $\alpha_{11}=\alpha_{22}=\alpha_{12}=1, \zeta^{*}=0, \gamma_{i}=1$, and $c_{1}=c_{2}=0$, so that the expression (26) becomes

$$
\eta^{*}=\frac{x_{1}^{2} R_{1}+x_{2}^{2} R_{2}+x_{1} x_{2} R_{12}}{x_{1}^{2} S_{1}+x_{2}^{2} S_{2}+x_{1} x_{2} S_{12}}
$$

where

$$
\begin{gathered}
R_{1}=\frac{2}{3}+\frac{2 \mu}{5}, \quad R_{2}=\frac{2}{3}+\frac{2}{5 \mu} \\
R_{12}=\frac{8}{15}+\frac{\sqrt{2}}{5}\left[\left(\frac{\sigma_{1}}{\sigma_{12}}\right)^{2} \mu_{21}^{-1 / 2} \mu_{12}^{-1}+\left(\frac{\sigma_{2}}{\sigma_{12}}\right)^{2} \mu_{12}^{-1 / 2} \mu_{21}^{-1}\right] \\
S_{1}=\frac{16}{5 \sqrt{2}} R_{1}\left(\frac{\sigma_{1}}{\sigma_{12}}\right)^{2} \mu_{21}^{1 / 2}, \quad S_{2}=\frac{16}{5 \sqrt{2}} R_{2}\left(\frac{\sigma_{2}}{\sigma_{12}}\right)^{2} \mu_{12}^{1 / 2} \\
S_{12}=\frac{32}{15}+\frac{16}{25}\left(\frac{\sigma_{1}}{\sigma_{12}}\right)^{2}\left(\frac{\sigma_{2}}{\sigma_{12}}\right)^{2}\left(\mu_{12} \mu_{21}\right)^{-1 / 2}
\end{gathered}
$$

Here, $\mu=\mu_{12} / \mu_{21}=m_{1} / m_{2}$ is the mass ratio. Equation (31) agrees with the results obtained in the first Sonine approximation to the coefficient of viscosity of a molecular gasmixture of hard spheres [21]. In the case of mechanically equivalent particles $\left(m_{1}=m_{2}\right.$, $\left.\alpha_{11}=\alpha_{22}=\alpha_{12} \equiv \alpha, \sigma_{1}=\sigma_{2} \equiv \sigma\right), \gamma_{i}=1, \zeta^{*}=(2 / 3)\left(1-\alpha^{2}\right)(1+3 c / 32)$, and

$$
c_{1}=c_{2}=c=\frac{32(1-\alpha)\left(1-2 \alpha^{2}\right)}{81-17 \alpha+30 \alpha^{2}(1-\alpha)}
$$


In this case, one gets

$$
\eta^{*}=\frac{15}{4}\left((1+\alpha)\left[(2+\alpha)+\frac{c}{128}(33 \alpha-39)\right]\right)^{-1} .
$$

This expression coincides with the one recently obtained for a heated granular monocomponent gas [4]. All this shows the self-consistency of the present description.

\section{MONTE CARLO SIMULATION FOR UNIFORM SHEAR FLOW WITH THERMOSTAT}

The USF is a nonequilibrium state characterized by constant partial densities $n_{i}$, a linear velocity profile $\mathbf{u}=\mathbf{u}_{i}=\mathbf{a} \cdot \mathbf{r}$, where the elements of the tensor a are $a_{k \ell}=a \delta_{k x} \delta_{\ell y}, a$ being the constant shear rate. In addition, the granular temperature $T$ and the pressure tensor $\mathrm{P}$ are uniform, while the mass and heat fluxes vanish by symmetry reasons. This special state is generated by Lees-Edwards boundary conditions [22] which are simple boundary periodic boundary conditions in the local Lagrangian frame $\mathbf{R} \equiv \mathbf{r}-\mathbf{a} \cdot \mathbf{r} t$ and $\mathbf{V} \equiv \mathbf{v}-\mathbf{a} \cdot \mathbf{r}$. In terms of these variables the velocity distribution functions are uniform [23]

$$
f_{i}(\mathbf{r}, \mathbf{v} ; t)=f_{i}(\mathbf{V} ; t)
$$

In the case of elastic collisions $(\zeta=0)$ and in the absence of a thermostatting force, the energy balance equation (11) yields the heating equation

$$
\partial_{t} T=-\frac{2}{3 n} a P_{x y}
$$

Since the granular temperature $T$ increases in time, so does the collision frequency $\nu(t)$ according to Eq. (25). As a consequence, the reduced shear rate $a^{*}(t)=a / \nu(t)$ (which is the relevant uniformity parameter) monotonically decreases with time and the system asymptotically tends towards that of (local) equilibrium. This implies that for sufficiently long times (which means here $a^{*} \ll 1$ ) the system reaches a regime described by linear hydrodynamics and the Navier-Stokes shear viscosity $\eta$ can be identified as $[14,15]$ 


$$
\frac{\nu}{n T} \eta=-\lim _{t \rightarrow \infty} \frac{P_{x y}^{*}}{a^{*}}
$$

where $P_{x y}^{*}=P_{x y} / n T$. This route has been shown to be quite efficient to measure the Navier-Stokes shear viscosity coefficient for dilute [24] and dense [15] gases.

For a granular mixture, unless a thermostat is introduced, the energy balance equation (11) leads to a steady state when the viscous heating effect is exactly balanced by the collisional cooling [25]. However, if the granular mixture is excited by the Gaussian thermostat

$$
\mathbf{F}_{i}^{\mathrm{th}}=\frac{1}{2} m_{i} \zeta \mathbf{V}
$$

that exactly compensates for the collisional energy loss, the viscous heating still heats the system and Eq. (39) remains valid. Consequently, the linear relationship (40) allows one to determine the shear viscosity coefficient in the long time limit. Recently, this idea has been used to measure the shear viscosity of a heated granular monocomponent gas [4]. The comparison with kinetic theory shows an excellent agreement over a wide range of values of the restitution coefficient. It must be noted that here $\eta$ represents the shear viscosity of an excited granular mixture and thus it does not necessarily coincide with the Navier-Stokes shear viscosity obtained in the unforced case [10]. As a matter of fact, the results obtained in Sec. III indicate that the transport properties are affected by the Gaussian thermostat and the expression (26) for the (reduced) shear viscosity differs from the one derived in the freely cooling case [10]. The use of thermostats to control collisional cooling in undriven systems is quite common $[12,26]$. Usually, the motivation is to produce a steady state while here is to remove the steady state in favor of one whose dynamics determines the viscosity.

The Boltzmann equation for a mixture of inelastic hard spheres under USF and subject to the external Gaussian force (41) reads:

$$
\partial_{t} f_{i}-a V_{y} \frac{\partial}{\partial V_{x}} f_{i}+\frac{1}{2} \zeta \frac{\partial}{\partial \mathbf{V}} \cdot\left(\mathbf{V} f_{i}\right)=\sum_{j} J_{i j}\left[\mathbf{V} \mid f_{i}(t), f_{j}(t)\right]
$$

The second term on the left-hand side represents an inertial force of the form $\mathbf{F}_{i}^{\text {in }}=-m_{i} \mathbf{a} \cdot \mathbf{V}$, while the third term represents the thermostat force $\mathbf{F}_{i}^{\text {th }}$ given by Eq. (41). Thus, in this 
frame, the system is in a homogenous state subjected to the action of the (total) force $\mathbf{F}_{i}^{\text {in }}+\mathbf{F}_{i}^{\text {th }}$. We have numerically solved Eq. (42) by means of the Direct Simulation Monte Carlo (DSMC) method [13]. This method was devised to mimic the processes involved in the Boltzmann collision term and its extension to deal with inelastic collisions is straightforward. In addition, since the USF is spatially homogeneous in the Lagrangian frame, the simulation method is easy to carry out and only the (peculiar) velocities of the particles need to be stored. The restriction to this homogeneous state prevents us from studying the possible formation of particle clusters (microstructure).

Technical details of the DSMC method and its application to the USF state can be found in Refs. [11] and [25]. In our simulations we have typically taken a total number of particles $N=10^{5}$, a number of replicas $\mathcal{N}=5$, and a time step $\Delta t=3 \times 10^{-3} \ell_{11} / v_{01}$, where $\ell_{11}=\left(\sqrt{2} \pi n_{1} \sigma_{1}^{2}\right)^{-1}$ is the mean free path for collisions $1-1$ and $v_{01}=\sqrt{2 T / m_{1}}$.

At given values of the shear rate $a$, the restitution coefficients $\alpha_{i j}$, and the parameters of the mixture, the system is initially prepared in a local equilibrium state with a temperature $T(0)=T_{0}$ such that the initial value of the reduced shear rate is $a_{0}^{*}=a / \nu\left(T_{0}\right)$. As the system evolves, we monitor the time evolution of the reduced shear rate $a^{*}(t)=a / \nu(T(t))$ and the reduced $x y$ element of the pressure tensor $P_{x y}^{*}(t)=P_{x y}(t) / n T(t)$. We observe that in all the cases, after a transient period, the ratio $\eta^{*} \equiv-P_{x y}^{*} / a^{*}$ reaches a constant value that is independent of the shear rate and time. This allows us to measure the corresponding shear viscosity coefficient $\eta$ as

$$
\eta(t)=\frac{n T(t)}{\nu(t)} \eta^{*}
$$

where the dimensionless shear viscosity $\eta^{*}$ is independent of time but depends on dissipation and the parameters of the mixture (masses, sizes and concentrations).

The theoretical prediction for $\eta^{*}$ can be obtained from the Chapman-Enskog solution to Eq. (42) up to first order in the shear rate $a$. In Appendix B it is easily proved that the first order solution to (42) leads to the same expression for the shear viscosity as the one obtained in Sec. III from the general Chapman-Enskog method specialized to USF. Thus, in 
the first Sonine approximation, the theoretical prediction of $\eta^{*}$ is given by Eqs. (26)-(30).

Before analyzing the dependence of the dimensionless shear viscosity coefficient $\eta^{*}$ on the parameters of the problem, it is instructive to test the consistency of the simulation method in the limit $a^{*} \rightarrow 0$ (which corresponds here to $t \nu \gg 1$ ). For long times and for given values of $\alpha, m_{1} / m_{2}, \sigma_{1} / \sigma_{2}$, and $n_{1} / n_{2}$, the reduced viscosity $\eta^{*}$ must reach a value independent of the inital preparation of the system. In Fig. 1, we plot the shear-rate dependent viscosities $\eta^{*}\left(a^{*}\right)$ measured in the simulation, relative to its Navier-Stokes value $\eta_{\mathrm{B}}^{*}$ given by the Boltzmann theory [Eqs. (26)-(30)] for three different choices of the initial shear rate $a_{0}^{*}: 0.2$, 0.3 , and 0.4 . Here, the restitution coefficient is $\alpha=0.9$, the mass ratio is $m_{1} / m_{2}=4$, the concentration ratio is $n_{1} / n_{2}=1$ and the size ratio is $\sigma_{1} / \sigma_{2}=3$. After a transient regime of a few mean free times, we observe that the curves corresponding to the three different initial conditions practically coincide. This means that a hydrodynamic regime independent of the inital conditions has been achieved. In addition, for very small values of $a^{* 2}$, the ratio $\eta^{*}\left(a^{*}\right) / \eta_{\mathrm{B}}^{*}$ fluctuate around 1 showing that in this regime the viscosity coefficient measured in the simulation is consistent with the value obtained from the Boltzmann kinetic theory. The same behavior has been found for other values of the restitution coefficient as well as of the parameters of the mixture. Notice that the limit $a^{*} \rightarrow 0$ is strictly unattainable in the USF because it requires an infinite amount of time. Also, the signal-to-noise ratio decreases in that limit so that the fluctuations increase.

\section{COMPARISON BETWEEN THEORY AND SIMULATION}

Once the consistency of the simulation method has been tested, we focus our attention on the study of transport properties in the Navier-Stokes regime. In this Section we compare the predictions of the Sonine approximation with the results obtained from the DSMC method. A complete presentation of the results is complex due to the high dimensionality of the parameter space: $\left\{\alpha_{11}, \alpha_{22}, \alpha_{12}, m_{1} / m_{2}, \sigma_{1} / \sigma_{2}, n_{1} / n_{2}\right\}$. For the sake of concreteness, henceforth we will assume that the spheres are made of the same material, i.e., $\alpha_{11}=\alpha_{22}=$ 
$\alpha_{12} \equiv \alpha$. This reduces the parameter space to four quantities.

Apart from the shear viscosity coefficient, another interesting quantity at this level of description is the temperature ratio $T_{1} / T_{2}$. This ratio measures the breakdown of the energy equipartition. The analysis of the temperature differences has been a subject of growing interest in the past few years among both theorists $[7,11,12,20]$ and experimentalists $[8,9]$. As was previously found from the Boltzmann kinetic theory [20], except for mechanically equivalent particles, the partial temperatures $T_{i}$ are different. For the sake of illustration, Fig. 2 shows the dependence of the temperature ratio on the size ratio $\sigma_{1} / \sigma_{2}$ for an equimolar mixture $\left(n_{1} / n_{2}=1\right)$ and three different values of the restitution coefficient $\alpha=0.9,0.8$, and 0.7. We consider a binary mixture of constant density and so, $m_{1} / m_{2}=\left(\sigma_{1} / \sigma_{2}\right)^{3}$. We observe that for large size ratios the temperature differences are quite important, even for moderate dissipation. It is also apparent that an excellent agreement between the theory (given by the first Sonine correction) and Monte Carlo simulations (symbols) is found over the entire range of values of size and mass ratios considered.

Next, we explore the influence of dissipation on the reduced shear viscosity $\eta^{*}(\alpha)$ for different values of the mass ratio, the size ratio, and the concentration ratio. Three different values of the (common) restitution coefficient are considered: $\alpha=0.9,0.8$, and 0.7 . In Fig. 3, we plot the ratio $\eta^{*}(\alpha) / \eta^{*}(1)$ versus the mass ratio $m_{1} / m_{2}$ for $\sigma_{1} / \sigma_{2}=n_{1} / n_{2}=1$. Here, $\eta^{*}(1)$ refers to the elastic value for the shear viscosity coefficient. Again, the symbols represent the simulation data while the lines refer to the theoretical results obtained from the Boltzmann equation in the first Sonine approximation. We see that in general the deviation of $\eta^{*}(\alpha)$ from its functional form for elastic collisions is quite important. This tendency becomes more significant as the mass disparity increases. The agreement between the first Sonine approximation and simulation is seen to be in general excellent. This agreement is similar to the one previously found in the monocomponent case [4]. At a quantitative level, the discrepancies between theory and simulation tend to increase as the restitution coefficient decreases, although these differences are quite small (say, for instance, around $2 \%$ at $\alpha=0.7$ in the disparate mass case $m_{1} / m_{2}=10$ ). 
The influence of the size ratio on the shear viscosity is shown in Fig. 4 for $m_{1} / m_{2}=4$ and $n_{1} / n_{2}=1$. We observe again a strong dependence of the shear viscosity on dissipation. However, for a given value of $\alpha$, the influence of $\sigma_{1} / \sigma_{2}$ on $\eta^{*}$ is weaker than the one found before in Fig. 3 for the mass ratio. The agreement for both $\alpha=0.9$ and $\alpha=0.8$ is quite good, except for the largest size ratio at $\alpha=0.8$. These discrepancies become more significant as the dissipation increases (say $\alpha=0.7$ ), especially for mixtures of particles of very different sizes. Finally, Fig. 5 shows the dependence of $\eta^{*}(\alpha) / \eta^{*}(1)$ on the concentration ratio for $m_{1} / m_{2}=4$ and $\sigma_{1} / \sigma_{2}=1$. We observe that both the theory and simulation predicts a very weak influence of composition on the shear viscosity. With respect to the influence of dissipation, the trends are similar to those of Figs. 3 and 4: the main effect of inelasticity in collisions is to enhance the momentum transport with respect to the case of elastic collisions. The agreement now between theory and simulation is very good, even for disparate values of the concentration ratio and/or strong dissipation. Therefore, according to the comparison carried out in Figs. 3, 4, and 5, we can conclude that the agreement extends over a wide range values of the restitution coefficient, indicating the reliability of the first Sonine approximation for describing granular flows beyond the quasielastic limit.

\section{DISCUSSION}

Although the utility of a hydrodynamic description for granular media under rapid flow

conditions has been recognized for many years, its domain of validity as well as the forms of the transport coefficients remain a topic of interest and controversy. In this context, there are some doubts about the possibility of going from a kinetic theory to a hydrodynamic level of description by using a Chapman-Enskog expansion around the homogenous cooling state. Given that the search for exact solutions of the Boltzmann equation is far beyond the present perspectives, an alternative to get some insight into the above question is to numerically solve the kinetic equation and compare these results with the corresponding solution obtained by assuming the validity of a hydrodynamic description. In this paper, we have performed such 
a comparison at the level of the shear viscosity coefficient of a heated granular mixture. The system is heated by the action of a thermostatting external force which exactly compensates for cooling effects associated with the inelasticity of collisions. Although some previous works $[4,27,28]$ have compared kinetic theory predictions for transport coefficients with computer simulations in the case of a monocomponent gas, studies for multicomponent granular gases are more scarce. Very recently, a seemingly similar analysis for the shear viscosity $\eta$ of a dense mixture has been given in Ref. [29]. Nevertheless, the above kinetic theory only holds for nearly elastic particles and the expression of $\eta$ in the first Sonine approximation coincides with the one obtained in the elastic case.

As a first step in our issue, in Sec. III we have derived the general hydrodynamic equations of a heated binary mixture of smooth inelastic spheres from the Boltzmann kinetic equation by using the Chapman-Enskog method. The corresponding transport coefficients have been expressed in terms of the solution to integral equations, which are then solved approximately (first Sonine polynomial approximation) just as in the case of elastic collisions. The explicit expressions for the set of relevant transport coefficients $\left\{D, D_{p}, D^{\prime}, D^{\prime \prime}, L, \lambda, \eta\right\}$ are displayed in Appendix A. In contrast to previous works [6,29], our results are not limited a priori to weak inelasticity and they take into account the effect of the temperature differences on the transport coefficients. On the other hand, the results obtained here for the transport coefficients slightly differ from those obtained in the freely cooling case [10], showing that in general the introduction of a thermostat affects the transport properties of the gas [23]. The Chapman-Enskog results obtained for the mixture have been then specialized to the hydrodynamic state of transverse shear. In this state, the shear viscosity coefficient $\eta$ is the relevant transport coefficient of the problem. The explicit form of $\eta$ is given by Eqs. (26)(30) in terms of the restitution coefficients $\alpha_{i j}$ and the parameters of the mixture (masses, diameters, and concentrations).

To test the assumptions of the Chapman-Enskog method and the approximate Sonine solution to the resulting integral equation, the DSMC method has been used to solve the Boltzmann equation in the uniform shear flow state. In the absence of a thermostat, in 
a granular fluid there is a competition between two opposite effects: viscous heating and collisional cooling. In that case, when both effects exactly cancel each other, a steady state is reached after a transient period. In this steady state, due to the coupling between dissipation and the shear rate, the system is far away from the Navier-Stokes regime, except when $\alpha \rightarrow 1$ [25]. However, if the external thermostat is adjusted to compensate for the energy lost in collisions, the shearing work still heats the system. As a consequence, as the system evolves, the reduced shear rate $a^{*}(t)$ goes to zero and the system achieves a regime described by linear hydrodynamics. In this regime, the Navier-Stokes shear viscosity coefficient can be measured from simulations. In this paper, the thermostat is used to remove the steady state in favor of a time-dependent state whose dynamics allows one to get the Navier-Stokes shear viscosity just as for the case of elastic collisions [15,24].

The dependence of the viscosity $\eta$ on the full parameter space has been explored. Specifically, the parameter space over which our solution has been verified is the mass ratio $m_{1} / m_{2}$, the concentration ratio $n_{1} / n_{2}$, the ratio of diameters $\sigma_{1} / \sigma_{2}$, and the (common) restitution coefficient $\alpha \equiv \alpha_{11}=\alpha_{11}=\alpha_{22}=\alpha_{12}$. The theory and simulation clearly show how in general, the influence of dissipation on momentum transport is quite important since there is a relevant dependence of the viscosity $\eta(\alpha)$ on the restitution coefficient $\alpha$. At a given value of the restitution coefficient, the dependence of $\eta(\alpha) / \eta(1)$ on the mass ratio is more significant than the one found on the composition and diameters. This feature has been also found for the temperature ratio in the experiments recently carried out in vibrated mixtures $[8,9]$, although experimental confirmation of the trends observed here for the viscosity is still lacking. With respect to the accuracy of the theory predictions we see that, in general, the Chapman-Enskog results in the first Sonine approximation exhibit an excellent agreement with the simulation data. This supports the idea that the Sonine polynomial approximation for granular fluids has an accuracy comparable to that for elastic collisions. Exceptions to this agreement are extreme mass ratios and strong dissipation. These discrepancies are due basically to the approximations introduced in applying the Chapman-Enskog method, and more specifically in using the first Sonine approximation. 
One of the main limitations of the results obtained here from the Boltzmann equation is its restriction to the low-density regime. In this regime, the collisional transfer contributions to the fluxes are negligible and only their kinetic contributions are taken into account. Possible extension in both aspects, theory and simulation, of the present simple hydrodynamic state to higher densities can be carried out in the context of the revised Enskog theory. In this case, many of the phenomena appearing in dense granular fluids (such as spontaneous formation of dense clusters surrounded by regions of low-density [30]) could be studied. On the other hand, although the comparison performed here has been made undergoing uniform shear flow without paying attention to the possible formation of particle clusters [31], our Chapman-Enskog results apply for general inhomogeneous situations. The only restriction is that they provide the irreversible parts of the mass, heat, and momentum fluxes to leading order in the spatial gradients of the hydrodynamic fields. In this context, the results derived in this paper can be used to analyze the behavior of granular mixtures in a lot of physical situations. Thus, for instance, the knowledge of the complete hydrodynamic equations for a binary mixture allows one to say whether the mixture hydrodynamics is more or less unstable to long-wavelength perturbations than that of the one-component case, and what are the mechanisms involved in phenomena very often observed in nature and experiments such as phase separation or segregation. We hope that the present results give some insight into the understanding of these interesting and complex problems.

\section{ACKNOWLEDGMENTS}

V. G. acknowledges partial support from the Ministerio de Ciencia y Tecnología (Spain) through Grant No. BFM2001-0718.

\section{APPENDIX A: CHAPMAN-ENSKOG EXPANSION}

In this Appendix, the expressions of the transport coefficients for a heated granular mixture are obtained. The derivation follows similar steps as those made in Ref. [10] in the 
free cooling case. Here, we will use the same notation as in Ref. [10]. In the first order, the distribution function $f_{i}^{(1)}$ verifies the kinetic equation

$$
\left(\mathcal{L}_{i}+\frac{1}{2} \zeta^{(0)} \frac{\partial}{\partial \mathbf{V}} \cdot \mathbf{V}\right) f_{i}^{(1)}+\mathcal{M}_{i} f_{j}^{(1)}=-\left(D_{t}^{(1)}+\mathbf{V} \cdot \nabla\right) f_{i}^{(1)}
$$

where $D_{t}^{(1)}=\partial_{t}^{(1)}+\mathbf{u} \cdot \nabla$, and

$$
\begin{gathered}
\mathcal{L}_{i} f_{i}^{(1)}=-\left(J_{i i}\left[f_{i}^{(0)}, f_{i}^{(1)}\right]+J_{i i}\left[f_{i}^{(1)}, f_{i}^{(0)}\right]+J_{i j}\left[f_{i}^{(1)}, f_{j}^{(0)}\right]\right), \\
\mathcal{M}_{i} f_{j}^{(1)}=-J_{i j}\left[f_{i}^{(0)}, f_{j}^{(1)}\right] .
\end{gathered}
$$

In these equations, it is understood that $i \neq j$ and use has been made of the fact that $\partial_{t}^{(0)} T=0$ and the results $\mathbf{j}_{i}^{(0)}=\mathbf{q}^{(0)}=\zeta^{(1)}=0$. The last equality follows from the fact that the cooling rate is a scalar, and so $\zeta^{(1)}$ should be proportional to $\nabla \cdot \mathbf{u}$. However, as shown later, there is no contribution to $f_{i}^{(1)}$ proportional to the divergence of the flow field so that $\zeta^{(1)}=0$ by symmetry. This property is special to the low density Boltzmann kinetic theory and such terms occur at higher densities [5]. The macroscopic balance equations to first order are

$$
D_{t}^{(1)} x_{1}=0, \quad \frac{3}{5} D_{t}^{(1)} \ln p=\frac{3}{2} D_{t}^{(1)} \ln T=-\nabla \cdot \mathbf{u}, \quad D_{t}^{(1)} \mathbf{u}=-\rho^{-1} \nabla p .
$$

Use of these in (A1) yields

$$
\left(\mathcal{L}_{i}+\frac{1}{2} \zeta^{(0)} \frac{\partial}{\partial \mathbf{V}} \cdot \mathbf{V}\right) f_{i}^{(1)}+\mathcal{M}_{i} f_{j}^{(1)}=\mathbf{A}_{i} \cdot \nabla x_{1}+\mathbf{B}_{i} \cdot \nabla p+\mathbf{C}_{i} \cdot \nabla T+D_{i, \alpha \beta} \nabla_{\alpha} u_{\beta},
$$

where

$$
\begin{gathered}
\mathbf{A}_{i}(\mathbf{V})=-\left(\frac{\partial}{\partial x_{1}} f_{i}^{(0)}\right)_{p, T} \mathbf{V}, \\
\mathbf{B}_{i}(\mathbf{V})=-\frac{1}{p}\left[f_{i}^{(0)} \mathbf{V}+\frac{n T}{\rho}\left(\frac{\partial}{\partial \mathbf{V}} f_{i}^{(0)}\right)\right], \\
\mathbf{C}_{i}(\mathbf{V})=\frac{1}{T}\left[f_{i}^{(0)}+\frac{1}{2} \frac{\partial}{\partial \mathbf{V}} \cdot\left(\mathbf{V} f_{i}^{(0)}\right)\right] \mathbf{V},
\end{gathered}
$$




$$
D_{i, \alpha \beta}(\mathbf{V})=V_{\alpha} \frac{\partial}{\partial V_{\beta}} f_{i}^{(0)}-\frac{1}{3} \delta_{\alpha \beta} \mathbf{V} \cdot \frac{\partial}{\partial \mathbf{V}} f_{i}^{(0)}
$$

The solutions to Eqs. (A5) are of the form

$$
f_{i}^{(1)}=\mathcal{A}_{i} \cdot \nabla x_{1}+\mathcal{B}_{i} \cdot \nabla p+\mathcal{C}_{i} \cdot \nabla T+\mathcal{D}_{i, \alpha \beta} \nabla_{\alpha} u_{\beta}
$$

The coefficients $\mathcal{A}_{i}, \mathcal{B}_{i}, \mathcal{C}_{i}$, and $\mathcal{D}_{i, \alpha \beta}$ are functions of the peculiar velocity $\mathbf{V}$ and the hydrodynamic fields. The cooling rate depends on space through its dependence on $x_{1}, p$, and $T$. The integral equations for the unknowns are easily identified as coefficients of the independent gradients in (A10). The result is

$$
\left(\mathcal{L}_{i}+\frac{1}{2} \zeta^{(0)} \frac{\partial}{\partial \mathbf{V}} \cdot \mathbf{V}\right)\left(\begin{array}{c}
\mathcal{A}_{i} \\
\mathcal{B}_{i} \\
\mathcal{C}_{i} \\
\mathcal{D}_{i, \alpha \beta}
\end{array}\right)+\mathcal{M}_{i}\left(\begin{array}{c}
\mathcal{A}_{j} \\
\mathcal{B}_{j} \\
\mathcal{C}_{j} \\
\mathcal{D}_{j, \alpha \beta}
\end{array}\right)=\left(\begin{array}{c}
\mathbf{A}_{i} \\
\mathbf{B}_{i} \\
\mathbf{C}_{i} \\
\mathbf{D}_{i, \alpha \beta}
\end{array}\right)
$$

Note that, in contrast to what happens in the free cooling case [10], here each one of the quantities $\mathcal{A}_{i}, \mathcal{B}_{i}, \mathcal{C}_{i}$, and $\mathcal{D}_{i, \alpha \beta}$ obey closed integral equations. The solution to Eq. (A11) provides the expression for the transport coefficients. In the case of the mass flux $\mathbf{j}_{1}$, these coefficients are identified as

$$
\begin{aligned}
& D=-\frac{\rho}{3 m_{2} n} \int d \mathbf{v} \mathbf{V} \cdot \mathcal{A}_{1}, \\
& D_{p}=-\frac{m_{1} p}{3 \rho} \int d \mathbf{v} \mathbf{V} \cdot \mathcal{B}_{1}, \\
& D^{\prime}=-\frac{m_{1} T}{3 \rho} \int d \mathbf{v} \mathbf{V} \cdot \mathcal{C}_{1} .
\end{aligned}
$$

The transport coefficients for the heat flux are

$$
\begin{gathered}
D^{\prime \prime}=-\frac{1}{3 T^{2}} \sum_{i=1}^{2} \int d \mathbf{v} \frac{1}{2} m_{i} V^{2} \mathbf{V} \cdot \mathcal{A}_{i}, \\
L=-\frac{1}{3} \sum_{i=1}^{2} \int d \mathbf{v} \frac{1}{2} m_{i} V^{2} \mathbf{V} \cdot \mathcal{B}_{i},
\end{gathered}
$$




$$
\lambda=-\frac{1}{3} \sum_{i=1}^{2} \int d \mathbf{v} \frac{1}{2} m_{i} V^{2} \mathbf{V} \cdot \mathcal{C}_{i} .
$$

Finally, the shear viscosity is given by

$$
\eta=-\frac{1}{10} \sum_{i=1}^{2} \int d \mathbf{v} m_{i} V_{\alpha} V_{\beta} \mathcal{D}_{i, \alpha \beta} .
$$

Accurate aproximations to the solutions to the integral equations for $\left(\mathcal{A}_{i}, \mathcal{B}_{i}, \mathcal{C}_{i}, \mathcal{D}_{i, \alpha \beta}\right)$ may be obtained using low order truncation of expansions in a series of Sonine polynomials. In the case of the mass flux, we consider the leading Sonine approximation (lowest degree polynomial)

$$
\left\{\mathcal{A}_{i}, \mathcal{B}_{i}, \mathcal{C}_{i}\right\} \rightarrow f_{i, M} \mathbf{V}\left\{a_{i, 1}, b_{i, 1}, c_{i, 1}\right\}, \quad f_{i, M}(\mathbf{V})=n_{i}\left(m_{i} / 2 \pi T_{i}\right)^{3 / 2} \exp \left(-m_{i} V^{2} / 2 T_{i}\right)
$$

where $a_{i, 1}=-\left(m_{1} m_{2} n / \rho n_{i} T_{i}\right) D, b_{i, 1}=-\left(\rho / p n_{i} T_{i}\right) D_{p}$, and $c_{i, 1}=-\left(\rho / T n_{i} T_{i}\right) D^{\prime}$. The coefficients $a_{i, 1}, b_{i, 1}$, and $c_{i, 1}$ are determined by multiplying the three first equations of Eq. (A11) by $m_{i} \mathbf{V}$ and integrating over the velocity. The result is

$$
\begin{gathered}
a_{1,1}=-\left(\nu_{D}-\frac{1}{2} \zeta^{(0)}\right)^{-1}\left(\frac{\partial}{\partial x_{1}} \ln n_{1} T_{1}\right)_{p, T}, \\
b_{1,1}=-\left(\nu_{D}-\frac{1}{2} \zeta^{(0)}\right)^{-1} \frac{n_{1} T_{1}}{p}\left(1-\frac{m_{1} n T}{\rho T_{1}}\right), \\
c_{1,1}=0 .
\end{gathered}
$$

Here, the collision frequency $\nu_{D}$ is given by Eq. (73) of Ref. [10].

In the case of the pressure tensor, the leading Sonine approximation for the function $\mathcal{D}_{i, \alpha \beta}$ is

$$
\mathcal{D}_{i, \alpha \beta} \rightarrow f_{i, M} d_{i, 1} R_{i, \alpha \beta}, \quad R_{i, \alpha \beta}=m_{i}\left(V_{\alpha} V_{\beta}-\frac{1}{3} V^{2} \delta_{\alpha \beta}\right)
$$

The shear viscosity coefficient is given by

$$
\eta=-n T^{2}\left(x_{1} \gamma_{1}^{2} d_{1,1}+x_{2} \gamma_{2}^{2} d_{2,1}\right)
$$


The coefficients $d_{i, 1}$ can be determined by multiplying the fourth equation of Eq. (A11) by $R_{i, \alpha \beta}$ and integrating over the velocity to get the coupled set of equations

$$
\left(\begin{array}{cc}
\tau_{11}-\zeta^{(0)} & \tau_{12} \\
\tau_{21} & \tau_{22}-\zeta^{(0)}
\end{array}\right)\left(\begin{array}{l}
d_{1,1} \\
d_{2,1}
\end{array}\right)=-\left(\begin{array}{c}
T_{1}^{-1} \\
T_{2}^{-1}
\end{array}\right) .
$$

The collision frequencies $\tau_{i j}=\tau_{i j}^{*} \nu$, where $\tau_{i j}^{*}$ are given by Eqs. (29) and (30). From Eq. (A25) one easily gets the expression (24) for the shear viscosity given in the main text.

The calculations for the heat flux are similar to those previously made for the other fluxes. As in the unforced case, this requires going to the second Sonine approximation. In this case, the transport coefficients defining the heat flux (16) are given by

$$
\begin{gathered}
D^{\prime \prime}=-\frac{5}{2} T\left(\frac{n_{1} \gamma_{1}^{3}}{m_{1}} a_{1,2}+\frac{n_{2} \gamma_{2}^{3}}{m_{2}} a_{2,2}\right)+\frac{5}{2} \frac{n m_{1} m_{2}}{\rho T}\left(\frac{\gamma_{1}}{m_{1}}-\frac{\gamma_{2}}{m_{2}}\right) D, \\
L=-\frac{5}{2} T^{3}\left(\frac{n_{1} \gamma_{1}^{3}}{m_{1}} b_{1,2}+\frac{n_{2} \gamma_{2}^{3}}{m_{2}} b_{2,2}\right)+\frac{5}{2} \frac{\rho}{n}\left(\frac{\gamma_{1}}{m_{1}}-\frac{\gamma_{2}}{m_{2}}\right) D_{p}, \\
\lambda=-\frac{5}{2} T^{3}\left(\frac{n_{1} \gamma_{1}^{3}}{m_{1}} c_{1,2}+\frac{n_{2} \gamma_{2}^{3}}{m_{2}} c_{2,2}\right)+\frac{5}{2} \rho\left(\frac{\gamma_{1}}{m_{1}}-\frac{\gamma_{2}}{m_{2}}\right) D^{\prime} .
\end{gathered}
$$

The coefficients $a_{i, 2}, b_{i, 2}$ and $c_{i, 2}$ obey the equations

$$
\begin{aligned}
& \left(\begin{array}{cc}
\nu_{11}-\frac{3}{2} \zeta^{(0)} & \nu_{12} \\
\nu_{21} & \nu_{22}-\frac{3}{2} \zeta^{(0)}
\end{array}\right)\left(\begin{array}{l}
a_{1,2} \\
a_{2,2}
\end{array}\right)=\left(\begin{array}{c}
X_{1} \\
X_{2}
\end{array}\right), \\
& \left(\begin{array}{cc}
\nu_{11}-\frac{3}{2} \zeta^{(0)} & \nu_{12} \\
\nu_{21} & \nu_{22}-\frac{3}{2} \zeta^{(0)}
\end{array}\right)\left(\begin{array}{l}
b_{1,2} \\
b_{2,2}
\end{array}\right)=\left(\begin{array}{c}
Y_{1} \\
Y_{2}
\end{array}\right), \\
& \left(\begin{array}{cc}
\nu_{11}-\frac{3}{2} \zeta^{(0)} & \nu_{12} \\
\nu_{21} & \nu_{22}-\frac{3}{2} \zeta^{(0)}
\end{array}\right)\left(\begin{array}{l}
c_{1,2} \\
c_{2,2}
\end{array}\right)=\left(\begin{array}{c}
Z_{1} \\
Z_{2}
\end{array}\right),
\end{aligned}
$$

where

$$
\begin{aligned}
X_{1}= & -\frac{\zeta^{(0)} m_{1} m_{2} n D}{\rho n_{1} T_{1}^{2}}-\frac{1}{2} \frac{T^{2}}{n_{1} T_{1}^{3}} \frac{\partial}{\partial x_{1}}\left(n_{1} \gamma_{1}^{2} c_{1}\right)+\frac{2}{15} \frac{m_{1}^{2} m_{2} n D}{\rho n_{1}^{2} T_{1}^{4}}\left[\int d \mathbf{v}_{1} \mathbf{S}_{1} \cdot \mathcal{L}_{1}\left(f_{1, M} \mathbf{V}_{1}\right)\right. \\
& \left.-\delta \gamma \int d \mathbf{v}_{1} \mathbf{S}_{1} \cdot \mathcal{M}_{1}\left(f_{2, M} \mathbf{V}_{2}\right)\right],
\end{aligned}
$$




$$
\begin{aligned}
Y_{1}= & -\frac{\zeta^{(0)} \rho D_{p}}{p n_{1} T_{1}^{2}}-\frac{1}{2} \frac{c_{1}}{p T_{1}}+\frac{2}{15} \frac{m_{1} \rho D_{p}}{p n_{1}^{2} T_{1}^{4}}\left[\int d \mathbf{v}_{1} \mathbf{S}_{1} \cdot \mathcal{L}_{1}\left(f_{1, M} \mathbf{V}_{1}\right)\right. \\
& \left.-\delta \gamma \int d \mathbf{v}_{1} \mathbf{S}_{1} \cdot \mathcal{M}_{1}\left(f_{2, M} \mathbf{V}_{2}\right)\right] \\
Z_{1}= & -\frac{\zeta^{(0)} \rho D^{\prime}}{T n_{1} T_{1}^{2}}-\frac{2+c_{1}}{2 T T_{1}}+\frac{2}{15} \frac{m_{1} \rho D^{\prime}}{T n_{1}^{2} T_{1}^{4}}\left[\int d \mathbf{v}_{1} \mathbf{S}_{1} \cdot \mathcal{L}_{1}\left(f_{1, M} \mathbf{V}_{1}\right)\right. \\
& \left.-\delta \gamma \int d \mathbf{v}_{1} \mathbf{S}_{1} \cdot \mathcal{M}_{1}\left(f_{2, M} \mathbf{V}_{2}\right)\right],
\end{aligned}
$$

and

$$
c_{i}=\frac{8}{15}\left[\frac{m_{i}^{2}}{4 n_{i} T_{i}^{2}} \int d \mathbf{v}_{1} V_{1}^{4} f_{i}^{(0)}-\frac{15}{4}\right]
$$

The corresponding expressions of the elements $X_{2}, Y_{2}$ and $Z_{2}$ can be deduced from Eqs. (A32), (A33) and (A34), respectively, by interchanging $1 \leftrightarrow 2$ and setting $D \rightarrow D, D_{p} \rightarrow$ $-D_{p}$ and $D^{\prime} \rightarrow-D^{\prime}$. The frequencies $\nu_{i j}$ and the collision integrals appearing in Eqs. (A31), (A32), (A33) and (A34) were explicitly evaluated in the Appendix D of Ref. [10]. Thus, the transport coefficients $D^{\prime \prime}, L$, and $\lambda$ are completely determined.

\section{APPENDIX B: FIRST ORDER SOLUTION TO THE USF}

In this Appendix we get the solution to Eq. (42) in the first order in the shear rate $a$. The normal solution to Eq. (42) is provided by the Chapman-Enskog method, i.e., a solution given as a power series in $a$ :

$$
f_{i}=f_{i}^{(0)}+f_{i}^{(1)}+\cdots
$$

The zeroth-order solution $f_{i}^{(0)}$ verifies Eq. (20) and it corresponds to the homogeneous cooling state distribution in the local Lagrangian frame. Its first Sonine approximation is given by Eq. (23). Inserting the expansion (B1) into Eq. (20) leads to the following integral equation for $f_{i}^{(1)}$ :

$$
\partial_{t} f_{i}^{(1)}-a V_{y} \frac{\partial}{\partial V_{x}} f_{i}^{(0)}+\frac{1}{2} \zeta^{(0)} \frac{\partial}{\partial \mathbf{V}} \cdot\left(\mathbf{V} f_{i}^{(1)}\right)+\frac{1}{2} \zeta^{(1)} \frac{\partial}{\partial \mathbf{V}} \cdot\left(\mathbf{V} f_{i}^{(0)}\right)=-\mathcal{L}_{i} f_{i}^{(1)}-\mathcal{M}_{i} f_{j}^{(1)}
$$


where the operators $\mathcal{L}_{i}$ and $\mathcal{M}_{i}$ are defined by Eqs. (A2) and (A3), respectively. Since $f_{i}^{(1)}$ depends on time only through the temperature, Eq. (39) implies that $\partial_{t} f_{i}^{(1)}=\mathcal{O}\left(a^{2}\right)$ and so the first term on the left hand side of Eq. (B1) vanishes in the first order. Further, $\zeta^{(1)}=0$ by symmetry because $\nabla \cdot \mathbf{u}=0$ in the USF. Taking into acount the above properties, Eq. (B2) reduces to

$$
\left(\mathcal{L}_{i}+\frac{1}{2} \zeta^{(0)} \frac{\partial}{\partial \mathbf{V}} \cdot \mathbf{V}\right) f_{i}^{(1)}+\mathcal{M}_{i} f_{j}^{(1)}=a V_{y} \frac{\partial}{\partial V_{x}} f_{i}^{(0)}
$$

This integral equation is identical to Eq. (A5) when one particularizes the latter one to USF. Therefore, the expression for the shear viscosity obtained from (B3) is given by Eqs. $(24)-(30)$. 


\section{REFERENCES}

[1] S. Chapman and T. G. Cowling, The Mathematical Theory of Nonuniform Gases (Cambridge University Press, Cambridge, 1970).

[2] J. J. Brey, J. W. Dufty, C. S. Kim, and A. Santos, Phys. Rev. E 58, 4638 (1998).

[3] J. J. Brey and D. Cubero, in Granular Gases, edited by T. Pöschel and S. Luding, Lecture Notes in Physics (Springer Verlag, Berlin, 2001), pp. 59-78.

[4] V. Garzó and J. M. Montanero, Physica A 313, 336 (2002).

[5] V. Garzó and J. W. Dufty, Phys. Rev. E 59, 5895 (1999).

[6] J. T. Jenkins and F. Mancini, Phys. Fluids A 1, 2050 (1989); P. Zamankhan, Phys. Rev. E 52, 4877 (1995); B. Arnarson and J. T. Willits, Phys. Fluids 10, 1324 (1998).

[7] S. R. Dahl, C. M. Hrenya, V. Garzó, and J. W. Dufty, Phys. Rev. E 66041301 (2002).

[8] R. D. Wildman and D. J. Parker, Phys. Rev. Lett. 88, 064301 (2002).

[9] K. Feitosa and N. Menon, Phys. Rev. Lett. 88, 198301 (2002).

[10] V. Garzó and J. W. Dufty, Phys. Fluids 14, 1476 (2002).

[11] J. M. Montanero and V. Garzó, Granular Matter 4, 17 (2002).

[12] A. Barrat and E. Trizac, Granular Matter 4, 57 (2002).

[13] G. A. Bird, Molecular Gas Dynamics and the Direct Simulation Monte Carlo of Gas Flows (Clarendon, Oxford, 1994).

[14] T. Naitoh and S. Ono, J. Chem. Phys. 70, 4515 (1979).

[15] J. M. Montanero and A. Santos, Phys. Rev. E 54, 438 (1996); Phys. Fluids 9, 2057 (1997).

[16] D. J. Evans and G. P. Morriss, Statistical Mechanics of Nonequilibrium Liquids (Aca- 
demic Press, London, 1990).

[17] C. Henrique, G. Batrouni, and D. Bideau, Phys. Rev. E 63, 011304 (2000).

[18] S. R. de Groot and P. Mazur, Nonequilibrium Thermodynamics (Dover, New York, 1984).

[19] D. N. Zubarev, Nonequilibrium Statistical Thermodynamics (Consultants Bureau, New York, 1974).

[20] V. Garzó and J. W. Dufty, Phys. Rev. E 60, 5706 (1999).

[21] See, for instance, S. Chapman and T. G. Cowling, The Mathematical Theory of Nonuniform Gases (Cambridge University Press, Cambridge, 1970), Eqs. (12.4,1)-(12.4,3), pp. 238-239.

[22] A. W. Lees and S. F. Edwards, J. Phys. C 5, 1921 (1972).

[23] J. W. Dufty, A. Santos, J. J. Brey, and R. F. Rodríguez, Phys. Rev. A 33, 459 (1986).

[24] J. Gómez Ordóñez, J. J. Brey, and A. Santos, Phys. Rev. A 39, 3038 (1989).

[25] J. M. Montanero and V. Garzó, Physica A 310, 17 (2002).

[26] D. R. M. Williams and F. C. MacKintosh, Phys. Rev. E 54, R9 (1996); T. P. C. van Noije, M. H. Ernst, E. Trizac, and I. Pagonabarraga, Phys. Rev. E 59, 4326 (1999); J. M. Montanero and A. Santos, Granular Matter 2, 53 (2000); S. J. Moon, M. D. Shattuck, and J. B. Swift, Phys. Rev. E 64, 031303 (2001).

[27] J. J. Brey, M. J. Ruiz-Montero, and D. Cubero, Europhys. Lett. 48, 359 (1999).

[28] C. Bizon, M. D. Shattuck, J. B. Swift, and H. L Swinney, Phys. Rev. E 60, 4340 (1999).

[29] M. Alam, J. T. Willits, B. Arnarson and S. Luding, Phys. Fluids 14, 4085 (2002).

[30] I. Goldhirsch and G. Zanetti, Phys. Rev. Lett. 70, 1619 (1993). 
[31] See, for instance, M. A. Hopkins and M. Y. Louge, Phys. Fluids A 3, 47 (1991); S. B. Savage, J. Fluid Mech. 241, 109 (1992); M. Babic, J. Fluid Mech. 254, 127 (1993); I. Goldhirsch, M.-L. Tan and G. Zanetti, J. Sci. Comput. 8, 1 (1993); P. J. Schmid and H. K. Kyötomaa, J. Fluid Mech. 264, 255 (1994). 


\section{FIGURES}

FIG. 1. Plot of the ratio $\eta^{*}\left(a^{*}\right) / \eta_{\mathrm{B}}^{*}$ as function of $a^{*}$ for $\alpha=0.9$ in the case $m_{1} / m_{2}=4$, $n_{1} / n_{2}=1$, and $\sigma_{1} / \sigma_{2}=3$ for three different values of the initial shear rate $a_{0}^{*}: a_{0}^{*}=0.2,0.3$, and 0.4. Here, $\eta_{\mathrm{B}}^{*}$ refers to the Navier- Stokes shear viscosity value given by the first Sonine approximation to the Boltzmann equation.

FIG. 2. Plot of the temperature ratio $T_{1} / T_{2}$ as a function of the size ratio $\sigma_{1} / \sigma_{2}=\left(m_{1} / m_{2}\right)^{1 / 3}$ for $n_{1} / n_{2}=1$ and three different values of the restitution coefficient $\alpha$ : (a) $\alpha=0.9$ (circles), (b) $\alpha=0.8$ (squares), and (c) $\alpha=0.7$ (triangles). The lines are the theoretical predictions and the symbols refer to the results obtained from the DSMC method.

FIG. 3. Plot of the ratio $\eta^{*}(\alpha) / \eta^{*}(1)$ as a function of the mass ratio $m_{1} / m_{2}$ for $\sigma_{1} / \sigma_{2}=n_{1} / n_{2}=1$ and three different values of the restitution coefficient $\alpha$ : (a) $\alpha=0.9$ (circles), (b) $\alpha=0.8$ (squares), and (c) $\alpha=0.7$ (triangles). The lines are the theoretical predictions and the symbols refer to the results obtained from the DSMC method.

FIG. 4. Plot of the ratio $\eta^{*}(\alpha) / \eta^{*}(1)$ as a function of the size ratio $\sigma_{1} / \sigma_{2}$ for $m_{1} / m_{2}=4$, $n_{1} / n_{2}=1$ and three different values of the restitution coefficient $\alpha$ : (a) $\alpha=0.9$ (circles), (b) $\alpha=0.8$ (squares), and (c) $\alpha=0.7$ (triangles). The lines are the theoretical predictions and the symbols refer to the results obtained from the DSMC method.

FIG. 5. Plot of the ratio $\eta^{*}(\alpha) / \eta^{*}(1)$ as a function of the concentration ratio $n_{1} / n_{2}$ for $m_{1} / m_{2}=4, \sigma_{1} / \sigma_{2}=1$ and three different values of the restitution coefficient $\alpha$ : (a) $\alpha=0.9$ (circles), (b) $\alpha=0.8$ (squares), and (c) $\alpha=0.7$ (triangles). The lines are the theoretical predictions and the symbols refer to the results obtained from the DSMC method. 


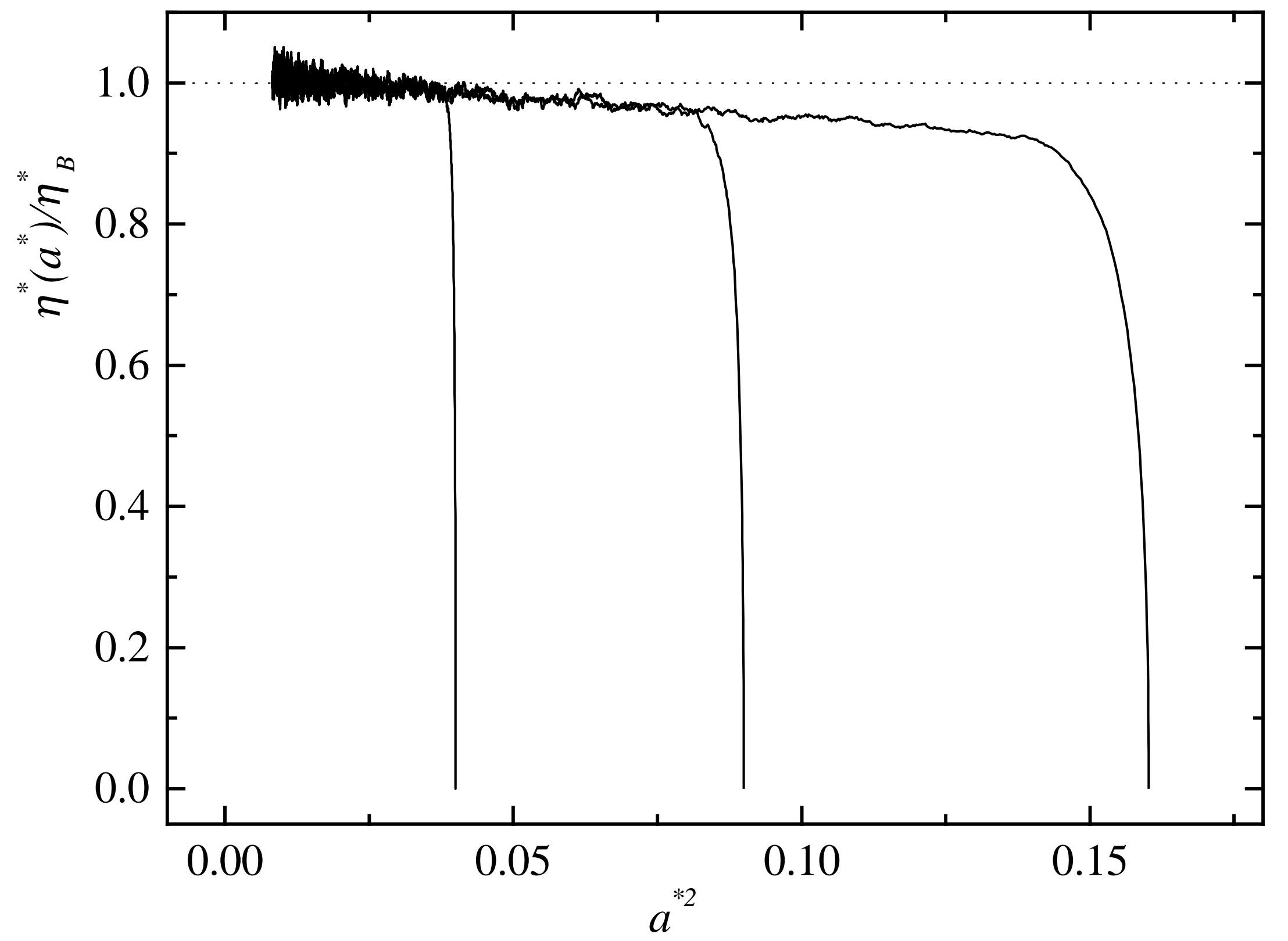




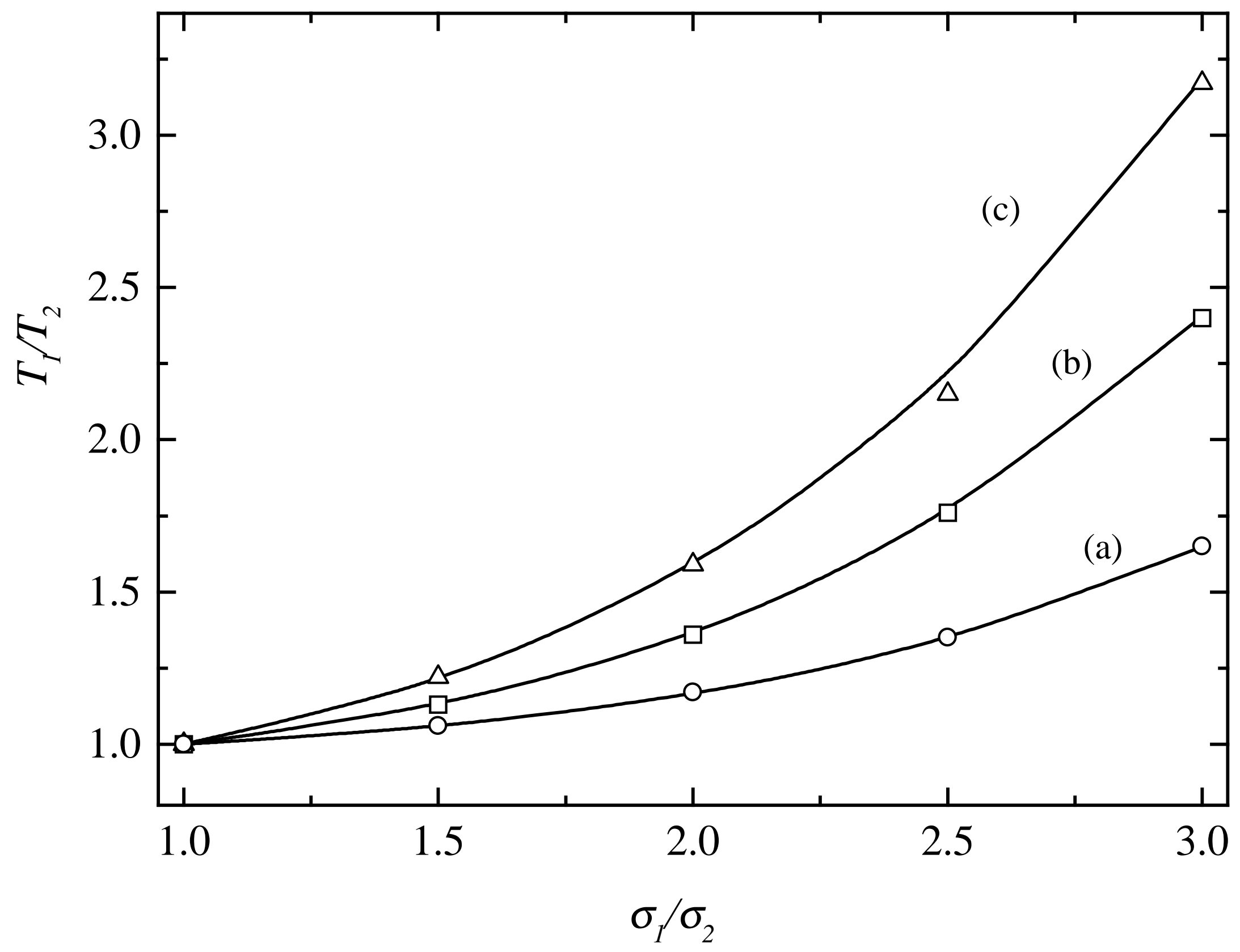




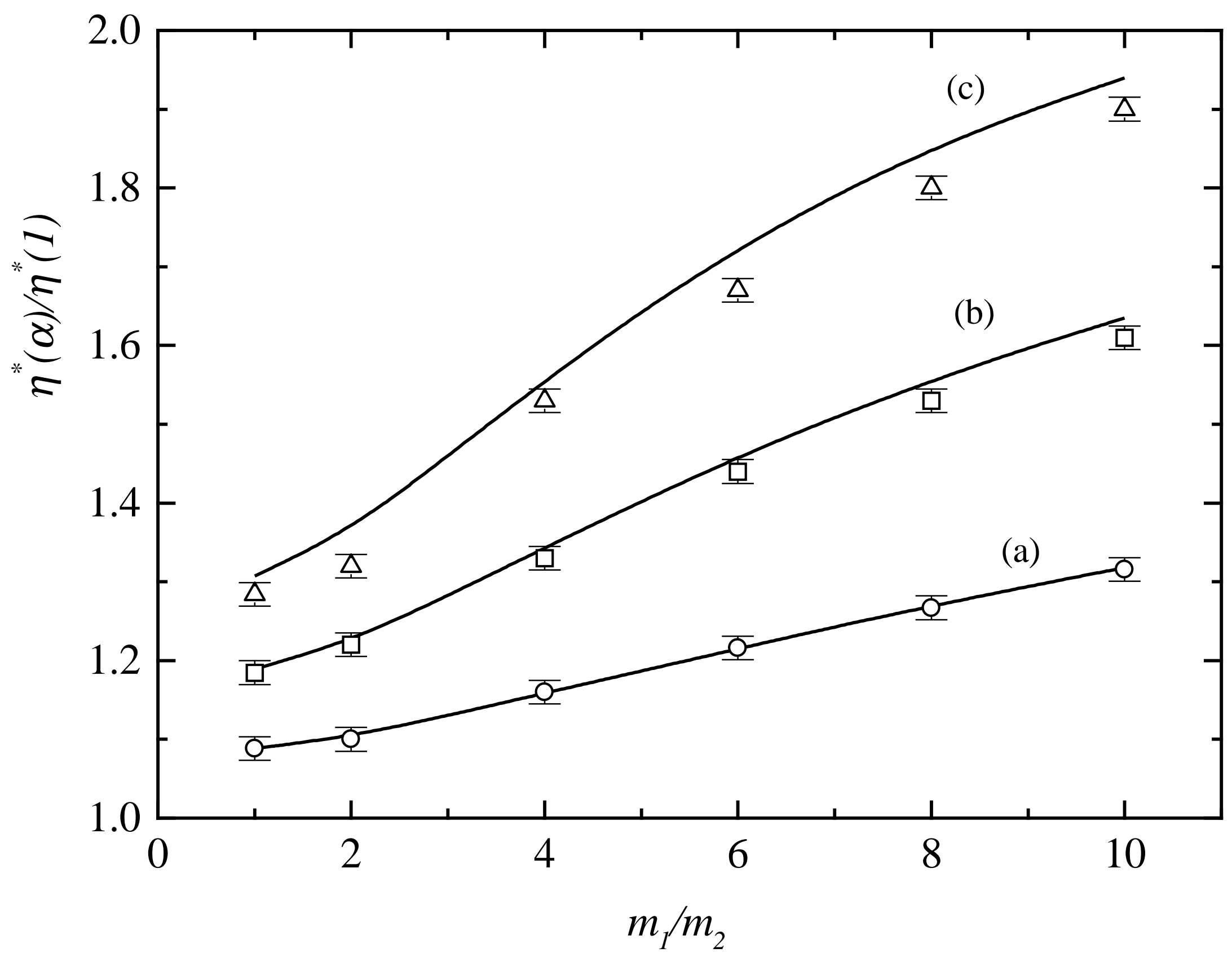




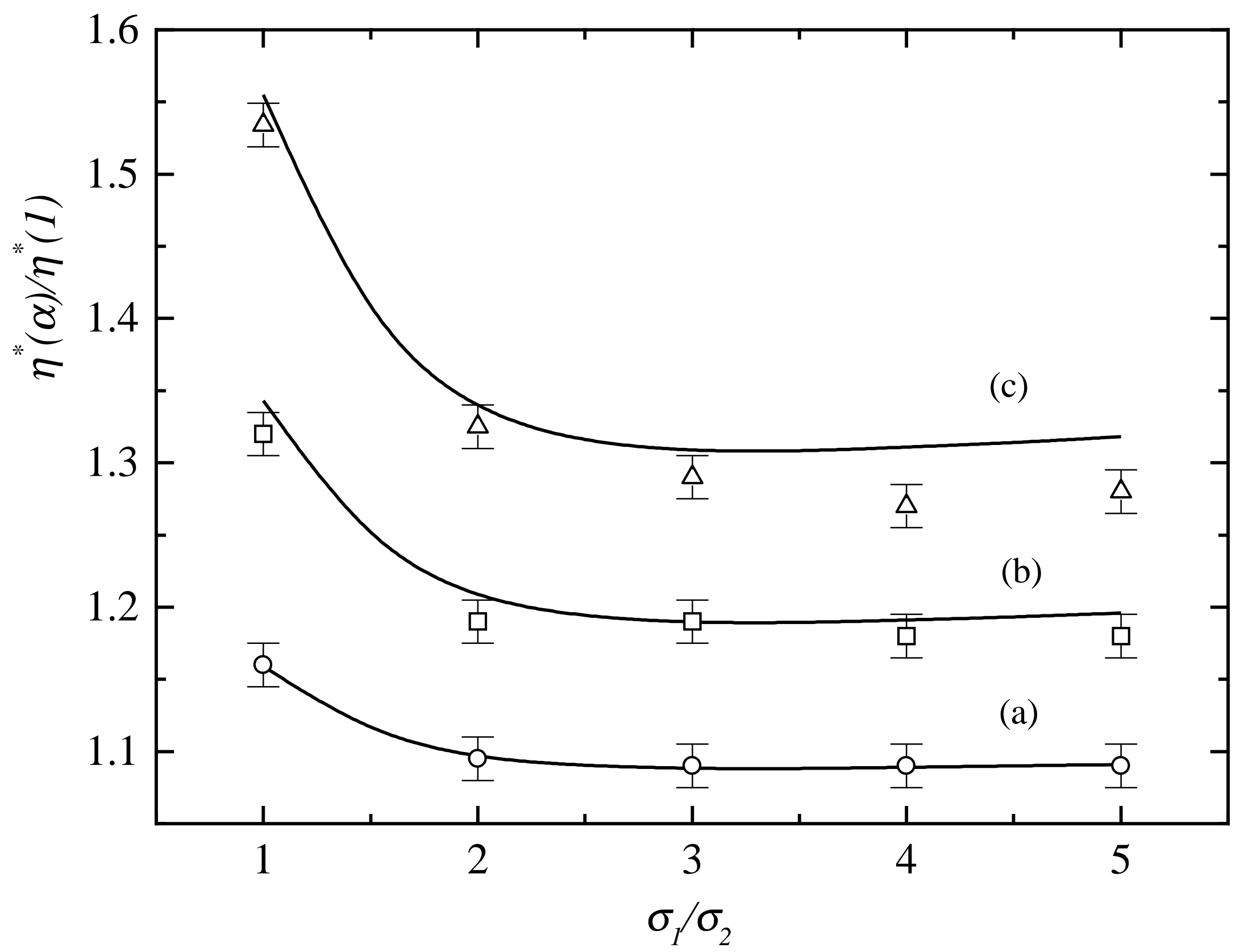




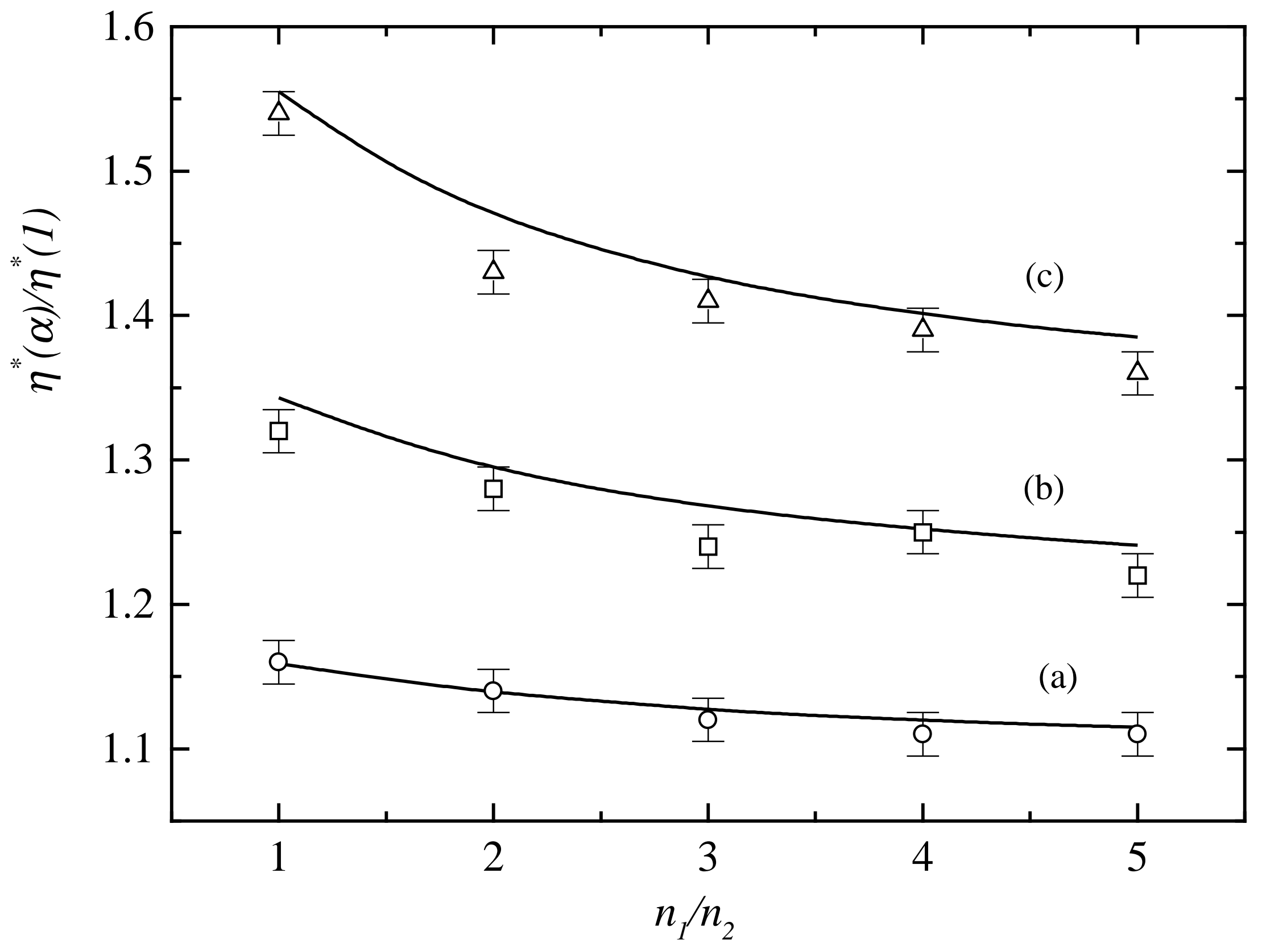

